\title{
openheart Relationship of age, atherosclerosis and angiographic stenosis using artificial intelligence
}

Rebecca Jonas (D) , ${ }^{1}$ James Earls, ${ }^{2}$ Hugo Marques, ${ }^{3}$ Hyuk-Jae Chang, ${ }^{4}$ Jung Hyun Choi, ${ }^{5}$ Joon-Hyung Doh, ${ }^{6}$ Ae-Young Her, ${ }^{7}$ Bon Kwon Koo, ${ }^{8}$ Chang-Wook Nam, ${ }^{9}$ Hyung-Bok Park, ${ }^{10}$ Sanghoon Shin, ${ }^{11}$ Jason Cole, ${ }^{12}$ Alessia Gimelli, ${ }^{13}$ Muhammad Akram Khan, ${ }^{14}$ Bin Lu, ${ }^{15}$ Yang Gao, ${ }^{16}$ Faisal Nabi, ${ }^{17}$ Ryo Nakazato, ${ }^{18} \mathrm{U}$ Joseph Schoepf, ${ }^{19}$ Roel S Driessen, ${ }^{20}$ Michiel J Bom, ${ }^{21}$ Randall C Thompson, ${ }^{22}$ James J Jang, ${ }^{23}$ Michael Ridner, ${ }^{24}$ Chris Rowan, ${ }^{25}$ Erick Avelar, ${ }^{26}$ Philippe Généreux, ${ }^{27}$ Paul Knaapen, ${ }^{28}$ Guus A de Waard (D) , ${ }^{28}$ Gianluca Pontone, ${ }^{29}$ Daniele Andreini, ${ }^{29}$ Mouaz H Al-Mallah, ${ }^{30}$ Robert Jennings, ${ }^{2}$ Tami R Crabtree, ${ }^{2}$ Todd C Villines, ${ }^{31}$ James K Min, ${ }^{2}$ Andrew D Choi ${ }^{32}$

- Additional supplemental material is published online only. To view, please visit the journal online (http://dx.doi.org/10. 1136/openhrt-2021-001832).

To cite: Jonas R, Earls J, Marques $\mathrm{H}$, et al. Relationship of age, atherosclerosis and angiographic stenosis using artificial intelligence. Open Heart 2021;8:e001832. doi:10.1136/ openhrt-2021-001832

Received 31 August 2021 Accepted 8 0ctober 2021

Check for updates

(c) Author(s) (or their employer(s)) 2021. Re-use permitted under CC BY-NC. No commercial re-use. See rights and permissions. Published by BMJ.

For numbered affiliations see end of article.

Correspondence to Dr Andrew D Choi; adchoi@mfa. gwu.edu

\section{ABSTRACT}

Objective The study evaluates the relationship of coronary stenosis, atherosclerotic plaque characteristics (APCs) and age using artificial intelligence enabled quantitative coronary computed tomographic angiography (Al-QCT).

Methods This is a post-hoc analysis of data from 303 subjects enrolled in the CREDENCE (Computed TomogRaphic Evaluation of Atherosclerotic Determinants of Myocardial IsChEmia) trial who were referred for invasive coronary angiography and subsequently underwent coronary computed tomographic angiography (CCTA). In this study, a blinded core laboratory analysing quantitative coronary angiography images classified lesions as obstructive $(\geq 50 \%)$ or non-obstructive $(<50 \%)$ while Al software quantified APCs including plaque volume (PV), low-density non-calcified plaque (LD-NCP), non-calcified plaque (NCP), calcified plaque (CP), lesion length on a per-patient and per-lesion basis based on CCTA imaging. Plaque measurements were normalised for vessel volume and reported as \% percent atheroma volume (\%PAV) for all relevant plaque components. Data were subsequently stratified by age $<65$ and $\geq 65$ years.

Results The cohort was $64.4 \pm 10.2$ years and $29 \%$ women. Overall, patients $>65$ had more PV and CP than patients $<65$. On a lesion level, patients $>65$ had more $\mathrm{CP}$ than younger patients in both obstructive $\left(29.2 \mathrm{~mm}^{3}\right.$ vs $48.2 \mathrm{~mm}^{3} ; p<0.04$ ) and non-obstructive lesions (22.1 $\mathrm{mm}^{3}$ vs $\left.49.4 \mathrm{~mm}^{3} ; \mathrm{p}<0.004\right)$ while younger patients had more \%PAV (LD-NCP) (1.5\% vs $0.7 \% ; p<0.038)$. Younger patients had more PV, LD-NCP, NCP and lesion lengths in obstructive compared with non-obstructive lesions. There were no differences observed between lesion types in older patients.

Conclusion Al-QCT identifies a unique APC signature that differs by age and degree of stenosis and provides a foundation for Al-guided age-based approaches to atherosclerosis identification, prevention and treatment.

\section{Key questions}

What is already known about this subject?

- High-risk atherosclerotic plaque characteristics put patients at risk for cardiovascular events, but is challenging for clinical practice using currently available methods.

What does this study add?

- This study provides a lesion-based assessment of plaque changes by age.

- This study provides support for use of artificial intelligence enabled quantitative coronary computed tomographic angiography (Al-QCT) to identify an age associated plaque phenotype.

How might this impact on clinical practice?

- These findings provide clinicians with an opportunity to tailor patient treatment based on the risk associated with a patient's plaque profile.

- These findings provide a basis for evaluating patients for enhanced prevention based on age with Al-QCT.

\section{INTRODUCTION}

Coronary computed tomographic angiography (CCTA) has been validated as a noninvasive imaging modality capable of both ruling out coronary artery disease (CAD) and quantifying coronary plaque. ${ }^{12}$ Advancements in the diagnostic specificity of CCTA have allowed not only for quantification of total plaque through the entirety of coronary vascular anatomy, but also for the specific identification and quantification of various plaque characteristics in stable and unstable lesions. ${ }^{3}$ This capability along with the use of artificial intelligence (AI) and machine 
learning have resulted in an expanding foundation of data describing high risk atherosclerotic plaque characteristics (APCs) that put specific patient populations at increased risk for major adverse cardiovascular events (MACE). ${ }^{4}$ Several APCs, namely, total plaque volume (PV), low density non-calcified plaque (LD-NCP), noncalcified plaque (NCP), calcified plaque (CP) and lesion length have been identified as significant quantifiable characteristics with prognostic value. ${ }^{56}$ The per cent atheroma volume (PAV) reflects each APC's quantity after normalising for vessel volume and has been suggested as a way of calculating and reporting APCs. Other significant quantifiable markers included vascular positive remodelling (PR), spotty calcification (SC), napkin-ring sign and high-risk plaque (HRP), defined as coronary lesions with both LD-NCP and PR. ${ }^{57-9}$ There have been distinct differences in the characteristics of these plaques based on various demographic and clinical variables, but whether these characteristics vary by age is still being investigated. This study intended to identify whether obstructive and non-obstructive lesions have different plaque characteristics based on age, specifically in individuals older than 65 compared with those younger than 65 years through plaque quantification using AI enabled quantitative coronary computed tomographic angiography (AI-QCT).

\section{METHODS}

The study population was comprised of the derivation cohort of the Computed TomogRaphic Evaluation of Atherosclerotic Determinants of Myocardial IsChEmia (CREDENCE) trial (ClinicalTrials.gov, NCT02173275), which was a prospective, multicentre diagnostic derivation-validation controlled clinical trial recruiting stable patients from 2014 to 2017. Sites and Investigators are listed in online supplemental appendix A. A detailed design manuscript was previously published. ${ }^{10}$ Enrolled patients underwent CCTA followed by invasive quantitative coronary angiography as the reference test within 60 days. Eligibility criteria included referral to nonemergent invasive coronary angiography (ICA) based on the American College of Cardiology/American Heart Association clinical practice guidelines for stable ischaemic heart disease. All index tests were interpreted in blinded fashion by an imaging core laboratory. Patient demographics, cardiovascular risk factors, laboratory values and medications were prospectively collected and recorded at the time of baseline and follow-up CCTAs.

\section{CCTA imaging protocols}

CCTA was performed using single or dual source CT scanners of $\geq 64$-detector rows. Sites performed CCTA in accordance with the guidelines established by the Society of Cardiovascular Computed Tomography (SCCT). ${ }^{11}$ Nitroglycerin was administered immediately prior to CCTA to enhance imaging quality. Image quality for CCTA was acceptable in $99 \%$ of patients.

\section{Al enabled quantification of CCTA}

The AI-based approach to CCTA interpretation in this study was performed using a validated and US Food and Drug Administration (FDA)-cleared software service (Cleerly Inc, New York, New York) that performs automated analysis of CCTA using a series of validated convolutional neural network models (including VGG19 network, 3D U-Net and VGG Network Variant) for image quality assessment, coronary segmentation and labelling, lumen wall evaluation and vessel contour determination and plaque characterisation. ${ }^{12}{ }^{13}$ The centerline algorithm was developed from 1007945 images, which comprised 23068 vessels from 3671 patients (online supplemental appendix B). The lumen and vessel wall algorithms were developed from 1414877 images, which comprised 8555 vessels from 3676 patients. First, the AI-aided approach produces a centerline along each coronary artery, and then for lumen and outer vessel wall contouring. This is applied to each phase of the examination and the two optimal series are identified for further analysis. After establishing vessel wall contours, distance and volumetric analyses were performed based on specified Hounsfield unit (HU) cut offs for characterising plaque characteristics. After the AI algorithm has finished all operations, as mandated by the FDA, a quality control cardiac CT trained technician reviews the results of the AI analysis in all cases with manual adjustment if necessary.

Coronary segments with a diameter $\geq 2 \mathrm{~mm}$ were included in the analysis using the modified 18-segment SCCT model. ${ }^{14}$ Each segment was evaluated for the presence or absence of coronary atherosclerosis, defined as any tissue structure $>1 \mathrm{~mm}^{2}$ within the coronary artery wall that was differentiated from the surrounding epicardial tissue, epicardial fat or the vessel lumen itself. The following APCs were evaluated:

- Atherosclerosis: Quantitative atherosclerosis characterisation was performed for every coronary artery and its branches using the automated AI-enabled web-based software platform (Cleerly Labs). ${ }^{13}$ PVs $\left(\mathrm{mm}^{3}\right)$ were calculated for each coronary lesion and then summated to compute the PV at the patient level. Plaque with a minimum volume of $\geq 3 \mathrm{~mm}^{3}$ was included for analysis. This provided data for analysis on both the lesion and patient level. PV was further categorised using $\mathrm{HU}$ ranges with NCP defined as 30-350 HU; LD-NCP defined as plaques <30 HU; and CP defined as $>350 \mathrm{HU}$. Coronary plaque burden was normalised to vessel volume to account for natural variation in coronary artery volume. Plaque burden was reported as PAV, CP PAV, NCP PAV and LD-NCP PAV which was calculated as PV (by each aforementioned type)/vessel volume $\times 100 \%$. Figure 1 provides an example of the software output for a patient with an obstructive left anterior descending (LAD) lesion predominantly comprised of NCP and LD-NCP. Figure 2 reflects the AI generated analysis of a non-obstructive LAD lesion comprised of NCP 


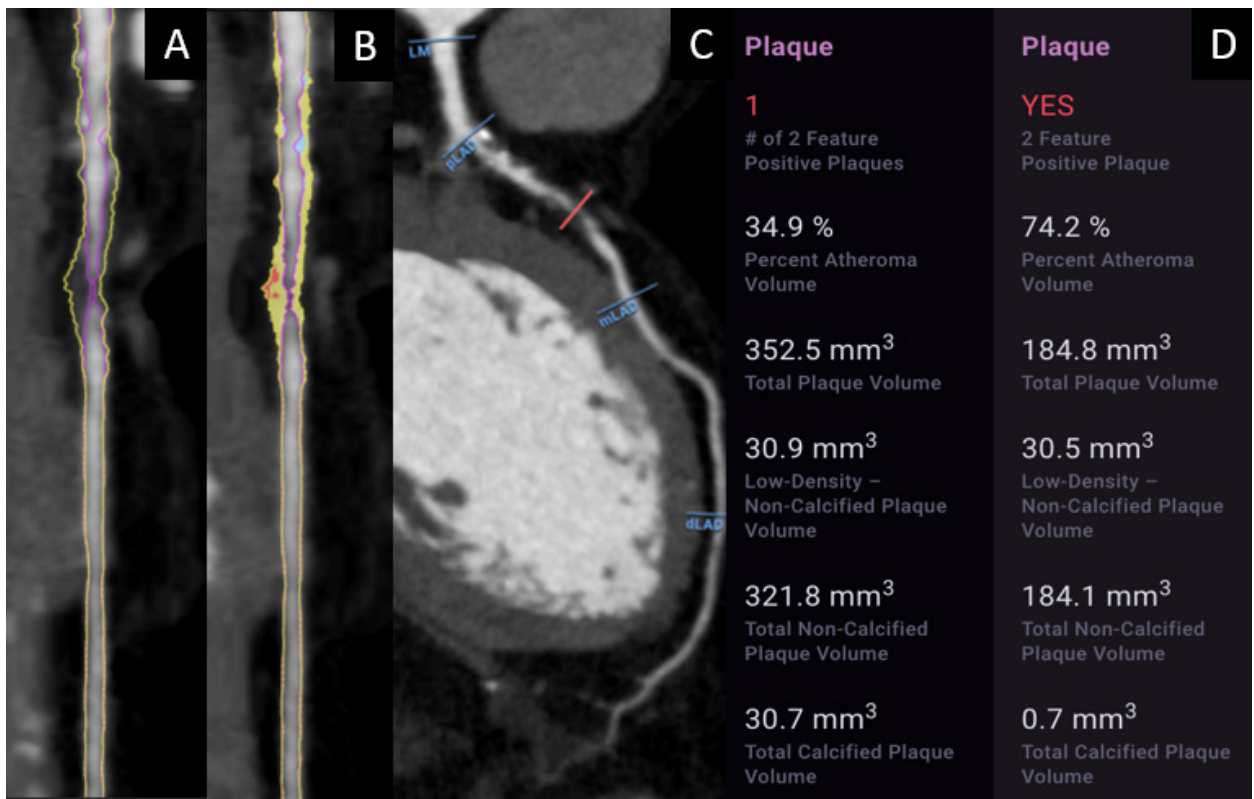

Figure 1 A 39-year-old with coronary CT angiography (CCTA) undergoing artificial intelligence (AI)-aided evaluation of stenosis and quantitative atherosclerosis burden. The patient demonstrates left anterior descending coronary artery obstructive stenosis (82\%) with a burden of plaque $\left(352.5 \mathrm{~mm}^{3}\right)$ consisting predominantly of non-calcified $\left(321.8 \mathrm{~mm}^{3}\right)$ that includes low-density non-calcified plaque (LD-NCP $30.5 \mathrm{~mm}^{3}$ ). (A) shows a CCTA straight reformat with plaque identified, while (B) shows a straight reformat with a colour overlay of non-calcified plaque (yellow), LD-NCP (red) and calcified plaque (blue). (C) shows a curved multiplanar reformat. (D) shows a graphical output of the quantified plaque volume by Al-aided evaluation. dLAD, distal left anterior descending; LM, left main; mLAD, mid left anterior descending; pLAD, proximal left anterior descending.

and LD-NCP while figure 3 provides an example of an right coronary artery (RCA) lesion comprised mostly of calcified plaque.
- Vascular remodelling: Arterial remodelling was calculated by examining the lesion diameter divided by the normal reference diameter. PR was defined as a ratio

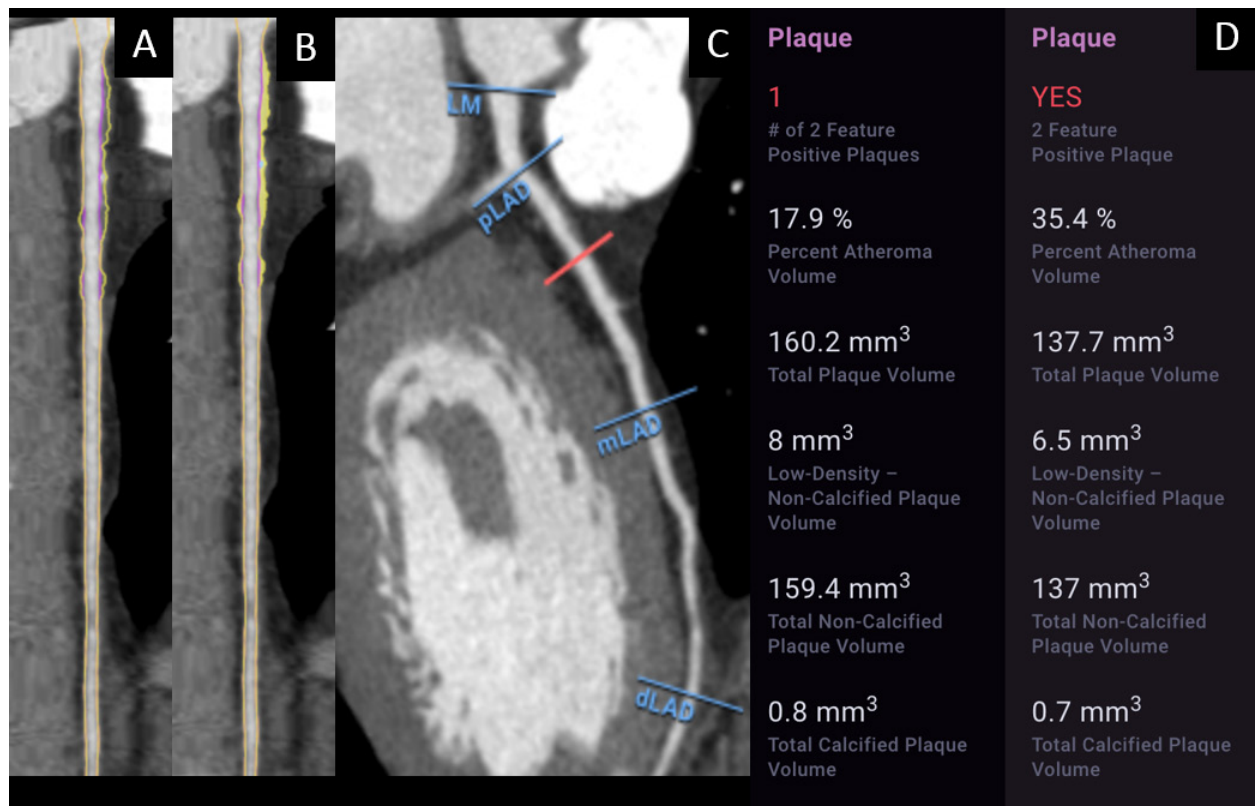

Figure 2 A 55-year-old with coronary CT angiography (CCTA) undergoing artificial intelligence (Al)-aided evaluation of stenosis and quantitative atherosclerosis burden. The patient demonstrates left anterior descending coronary artery non-obstructive stenosis $(25 \%)$ with a burden of plaque $\left(160.2 \mathrm{~mm}^{3}\right)$ consisting predominantly of non-calcified $\left(159.4 \mathrm{~mm}^{3}\right)$ that includes nonnegligible low-density non-calcified plaque $\left(8 \mathrm{~mm}^{3}\right)$. (A) shows a CCTA straight reformat with plaque identified, while (B) shows a straight reformat with a colour overlay of non-calcified plaque (yellow). (C) shows a curved multiplanar reformat. (D) shows a graphical output of the quantified plaque volume by Al-aided evaluation. dLAD, distal left anterior descending; LM, left main; $\mathrm{mLAD}$, mid left anterior descending; pLAD, proximal left anterior descending. 


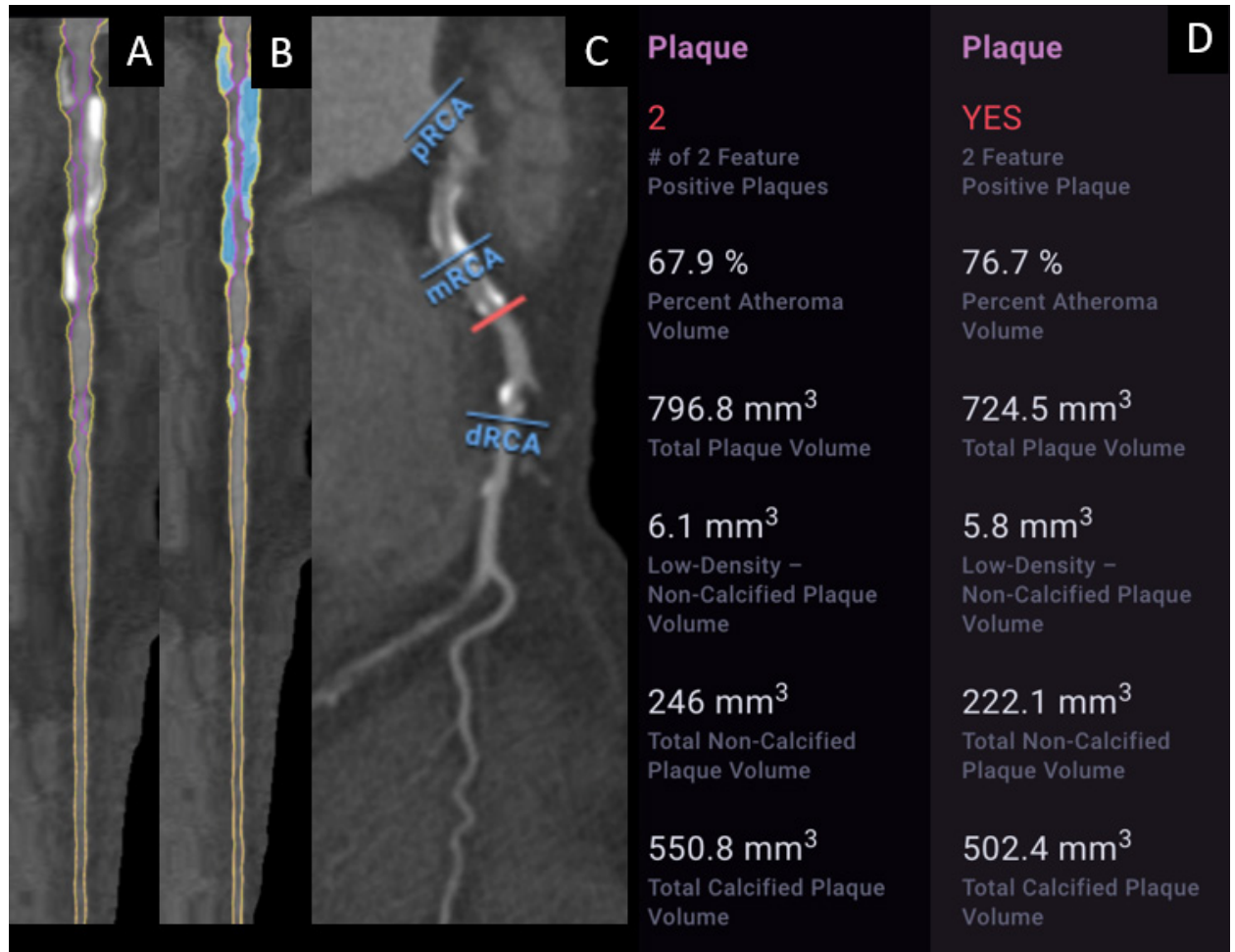

Figure 3 A 74-year-old with coronary CT angiography (CCTA) undergoing artificial intelligence (AI)-aided evaluation of stenosis and quantitative atherosclerosis burden. The patient demonstrates right coronary artery obstructive stenosis (61\%) with a high burden of plaque $\left(796.8 \mathrm{~mm}^{3}\right)$ consisting predominantly of calcified plaque $\left(550.8 \mathrm{~mm}^{3}\right)$. (A) shows a CCTA straight reformat with plaque identified, while (B) shows a straight reformat with a colour overlay of non-calcified plaque (yellow), and calcified plaque (blue). (C) shows a curved multiplanar reformat. (D) shows a graphical output of the quantified plaque volume by Alaided evaluation. dLAD, distal left anterior descending; LM, left main; mLAD, mid left anterior descending; pLAD, proximal left anterior descending.

$\geq 1.10$, negative remodelling was defined as a ratio of $<0.95$ and intermediate remodelling was a ratio between 0.95 and $1.10 .^{15}$

- HRP: HRPs were defined as coronary lesions with both LD-NCP and PR. ${ }^{7}$

- Other APCs: Plaque length measured uninterrupted plaque along the length of a vessel. Plaque diffusivity was the percent plaque along the length of a vessel divided by the total vessel length. ${ }^{16}$

\section{Quantitative coronary angiography}

Quantitative coronary angiography (QCA) was performed by a blinded core laboratory using an automated edgedetection algorithm by standard approaches as previously reported. ${ }^{17}$ Angiographic percent diameter stenosis, and lumen diameters of the proximal and distal reference segments were measured.

\section{Statistical analysis}

All statistical analyses were performed using SAS V.9.4 (SAS, Cary, North Carolina). Continuous data are reported as mean $\pm \mathrm{SD}$, and categorical variables are presented as absolute numbers with corresponding frequencies. Student's t-test, Mann-Whitney test, $\chi^{2}$ and Fisher exact tests were used to compare the distribution of continuous and categorical variables, respectively.

\section{Patient and public involvement statement}

This is a retrospective study that analyses prospectively collected de-identified multicentre data from the CREDENCE trial. Patients were recruited and consented for participation in the CREDENCE trial, but were not interactive with investigators conducting the substudy analysis. However, patients were involved in the design of the study to the extent that their invasive and noninvasive imaging was used to conduct this substudy, and study findings are aimed at improving clinical knowledge of cardiac disease in their cohort.

\section{RESULTS}

The study cohort was comprised of 303 patients whose mean age was $64.4 \pm 10.2$ years. The cohort was $29 \%$ women, $71 \%$ Asian and had a high prevalence of CAD risk factors including $64 \%$ with hypertension, $45 \%$ with dyslipidaemia and $48 \%$ with prior tobacco use (table 1). Nearly half of the patients (48\%) demonstrated typical or atypical angina, while over one-third $(35 \%)$ were asymptomatic. When stratifying by QCA there were significantly more women with non-obstructive $(<50 \%)$ CAD (39\% vs $22 \% \mathrm{p}=0.001)$. Patients with obstructive stenosis $(\geq 50 \%)$ were significantly more likely than patients with non-obstructive stenosis to have a family history of $\mathrm{CAD}$ and to have used tobacco, with 
Table 1 Baseline demographics

\begin{tabular}{|c|c|c|c|c|}
\hline & All $(n=303)$ & $\begin{array}{l}\text { Non-obstructive } \\
(<50 \%)(n=128)\end{array}$ & $\begin{array}{l}\text { Obstructive }(\geq 50 \%) \\
(n=175)\end{array}$ & $P$ value \\
\hline Age, years, mean (SD) & $64.4(10.2)$ & $64.3(10.2)$ & $64.6(10.2)$ & 0.8 \\
\hline Hypertension & $195(64 \%)$ & $79(62 \%)$ & $116(66 \%)$ & 0.41 \\
\hline Dyslipidaemia & $135(45 \%)$ & $56(44 \%)$ & $79(45 \%)$ & 0.81 \\
\hline Tobacco use & $146(48 \%)$ & $53(41 \%)$ & $93(53 \%)$ & 0.04 \\
\hline \multicolumn{5}{|l|}{ Symptoms } \\
\hline Typical angina & $109(36 \%)$ & $36(28 \%)$ & $73(42 \%)$ & 0.11 \\
\hline Atypical & $49(16 \%)$ & $23(18 \%)$ & $26(15 \%)$ & \\
\hline
\end{tabular}

no differences noted for prevalence of hypertension, dyslipidaemia or diabetes (table 2 ). Among younger (age <65) versus older patients (age $\geq 65$ ), hypertension was more commonly observed in older than younger patients $(74 \%$ vs $53 \%$ ). There were no significant differences in gender composition, dyslipidaemia, diabetes or symptoms between younger and older individuals.

Table 3 summarises CCTA findings evaluated by AI as a function of non-obstructive $(<50 \%)$ and obstructive $(\geq 50 \%)$ stenosis by QCA for patients $<65$ and $\geq 65$ on a per-patient basis. In patients with both non-obstructive $(<50 \%)$ and obstructive stenosis, patients $\geq 65$ had overall significantly greater plaque volume, total plaque $\% \mathrm{PAV}$, and calcified plaque when compared with patients $<65$. In examining specific APCs, older patients had overall higher PV (792.7 $\pm 486.1 \mathrm{~mm}^{3}$ vs $\left.500.1+349.8 \mathrm{~mm}^{3} ; \mathrm{p}<0.0001\right)$ across plaque components including higher calcified plaque $\left(366.5 \pm 336.2 \mathrm{~mm}^{3}\right.$ vs $\left.148.0 \pm 187.5 \mathrm{~mm}^{3} ; \mathrm{p}<0.0001\right)$ and calcified \% PAV ( $4.6 \%$ vs $2.6 \% ; \mathrm{p}=0.01)$. However, younger patients with non-obstructive disease demonstrated a similar degree of NCP $\left(243.0 \pm 220.2 \mathrm{~mm}^{3}\right.$ vs $286.5 \pm 190.2$ $\left.\mathrm{mm}^{3} ; \mathrm{p}=\mathrm{NS}\right)$ and LD-NCP $\left(8.6 \pm 11.1 \mathrm{~mm}^{3}\right.$ vs $8.9 \pm 12.9 \mathrm{~mm}^{3}$; $\mathrm{p}=\mathrm{NS}$ ) when compared with older patients. Obstructive lesions in patients $>65$ had higher PAV of calcified $(8.1 \%$ vs $3.2 \%$; $\mathrm{p}<0.0001)$; and NCP $(9.1 \%$ vs $7.6 \%$; $=0.007)$ than younger patients with obstructive lesions (table 3 ).

Further evaluating APCs on an individual lesion basis, table 4 shows the PV by AI-QCT of individual nonobstructive lesions was greater in patients $\geq 65$ versus patients $<65\left(105.6 \mathrm{~mm}^{3}\right.$ vs $\left.60.5 \mathrm{~mm} 3 ; \mathrm{p}<0.005\right)$. However, non-obstructive lesions in patients $<65$ exhibit a greater proportion of $\%$ PAV (LD-NCP) $(1.5 \%$ vs $0.7 \%$; $=0.038)$,

Table 2 Demographics of patients with non-obstructive stenoses and obstructive stenosis by age

\begin{tabular}{|c|c|c|c|c|c|c|c|c|c|}
\hline \multirow[b]{2}{*}{ Variable, n (\%) } & \multirow[b]{2}{*}{$\begin{array}{l}\text { Age <65 } \\
(\mathrm{N}=139)\end{array}$} & \multirow[b]{2}{*}{$\begin{array}{l}\text { Age } \geq 65 \\
(N=164)\end{array}$} & \multirow[b]{2}{*}{$P$ value } & \multicolumn{3}{|c|}{ Non-obstructive $(<50 \%)(\mathrm{N}=128)$} & \multicolumn{3}{|c|}{ Obstructive ( $\geq 50 \%)(\mathrm{N}=175)$} \\
\hline & & & & $\begin{array}{l}\text { Age <65 } \\
(\mathrm{N}=62)\end{array}$ & $\begin{array}{l}\text { Age } \geq 65 \\
(N=66)\end{array}$ & $P$ value & $\begin{array}{l}\text { Age }<65 \\
(N=77)\end{array}$ & $\begin{array}{l}\text { Age } \geq 65 \\
(\mathrm{~N}=98)\end{array}$ & $P$ value \\
\hline $\begin{array}{l}\text { Age, years, mean } \\
\text { (SD) }\end{array}$ & $55.7(7)$ & $71.9(5)$ & $<0.001$ & $56.1(7)$ & $71.9(6)$ & $<0.0001$ & $55.3(7)$ & $71.8(5)$ & $<0.0001$ \\
\hline Female & $36(26)$ & $52(32)$ & 0.27 & $23(37)$ & $27(41)$ & 0.66 & $13(17)$ & $25(26)$ & 0.17 \\
\hline Hypertension & $74(53)$ & $121(74)$ & $<0.0001$ & $33(53)$ & $46(70)$ & 0.05 & $41(53)$ & $75(77)$ & 0.001 \\
\hline Dyslipidaemia & $56(40)$ & $79(48)$ & 0.17 & $24(39)$ & $32(49)$ & 0.26 & $32(42)$ & $47(48)$ & 0.40 \\
\hline Diabetes & $38(27)$ & $57(35)$ & 0.17 & $17(27)$ & $21(32)$ & 0.59 & $21(27)$ & $36(37)$ & 0.18 \\
\hline Family history & $35(25)$ & $24(15)$ & 0.02 & $13(21)$ & $5(8)$ & 0.03 & $22(29)$ & $19(19)$ & 0.15 \\
\hline Tobacco use & $75(54)$ & $71(43)$ & 0.06 & $26(42)$ & $27(41)$ & 0.90 & $49(64)$ & $44(45)$ & 0.01 \\
\hline Symptoms & & & 0.31 & & & 0.95 & & & 0.06 \\
\hline Typical angina & $52(37)$ & $57(35)$ & & $18(29)$ & $18(27)$ & & $34(44)$ & $39(40)$ & \\
\hline Atypical & $25(18)$ & $24(14)$ & & $10(16)$ & $13(20)$ & & $15(20)$ & $11(11)$ & \\
\hline Non-cardiac & $13(9)$ & 27 (16) & & $9(15)$ & $10(15)$ & & $4(5)$ & $17(17)$ & \\
\hline Asymptomatic & $49(32)$ & $56(34)$ & & $25(40)$ & $25(38)$ & & $24(31)$ & $31(32)$ & \\
\hline
\end{tabular}


Table 3 Per-patient adverse plaque characteristics by artificial intelligence enabled quantitative coronary computed tomographic angiography by non-obstructive versus obstructive angiographic stenosis stratified by age

\begin{tabular}{|c|c|c|c|c|c|c|}
\hline \multirow[b]{2}{*}{ Variable, mean (SD) } & \multicolumn{3}{|c|}{$\begin{array}{l}\text { Non-obstructive }(<50 \%) \text { per-patient } \\
(\mathrm{N}=128)\end{array}$} & \multicolumn{3}{|c|}{$\begin{array}{l}\text { Obstructive ( } \geq 50 \%) \text { per-patient } \\
(\mathrm{N}=175)\end{array}$} \\
\hline & $\begin{array}{l}\text { Age }<65 \\
(\mathrm{~N}=62)\end{array}$ & $\begin{array}{l}\text { Age } \geq 65 \\
(\mathrm{~N}=66)\end{array}$ & $P$ value & $\begin{array}{l}\text { Age }<65 \\
(\mathrm{~N}=77)\end{array}$ & $\begin{array}{l}\text { Age } \geq 65 \\
(\mathrm{~N}=98)\end{array}$ & $P$ value \\
\hline $\mathrm{PV}, \mathrm{mm}^{3}$ & $357.5(379.3)$ & $510.7(206.2)$ & 0.02 & $500.1(349.8)$ & $792.7(486.1)$ & $<0.0001$ \\
\hline LD-NCP, $\mathrm{mm}^{3}$ & $8.6(11.1)$ & $8.9(12.9)$ & 0.46 & $15.0(15.3)$ & $12.4(15.8)$ & 0.11 \\
\hline $\mathrm{NCP}, \mathrm{mm}^{3}$ & 243.0 (220.2) & $286.5(190.2)$ & 0.10 & $352.1(266.8)$ & $426.2(262.7)$ & 0.02 \\
\hline $\mathrm{CP}, \mathrm{mm}^{3}$ & $114.5(190.5)$ & $224.2(372.1)$ & 0.007 & $148.0(187.5)$ & $366.5(336.2)$ & $<0.0001$ \\
\hline Total plaque \%PAV & $8.2(7.3)$ & $11.1(8.6)$ & 0.03 & $10.7(6.9)$ & $17.2(9.7)$ & $<0.0001$ \\
\hline LD-NCP \%PAV & $0.2(0.2)$ & $0.2(0.3)$ & 0.41 & $0.3(0.3)$ & $0.2(0.2)$ & 0.07 \\
\hline NCP \%PAV & $5.6(4.4)$ & $6.5(4.0)$ & 0.14 & $7.6(5.1)$ & $9.1(4.7)$ & 0.007 \\
\hline CP \%PAV & $2.6(3.6)$ & $4.6(6.2)$ & 0.01 & $3.2((4.0)$ & $8.1(7.0)$ & $<0.0001$ \\
\hline$\%$ plaque calcified & $25.3(21.3)$ & $33.9(22.4)$ & 0.21 & $26.6(21.3)$ & $42.5(20.5)$ & $<0.0001$ \\
\hline Remodelling index & $1.30(0.20)$ & $1.35(0.21)$ & 0.50 & $1.38(0.23)$ & $1.40(0.22)$ & 0.44 \\
\hline Positive remodelling $>1.1, n(\%)$ & $48(79)$ & $55(83)$ & 0.56 & $67(88)$ & $88(90)$ & 0.73 \\
\hline Intermediate remodelling, $\mathrm{n}(\%)$ & $10(16)$ & $10(15)$ & & $9(12)$ & $10(10)$ & \\
\hline Negative remodelling, $\mathrm{n}(\%)$ & $3(5)$ & $1(2)$ & & 0 & 0 & \\
\hline HRP (LD-NCP +PR), n (\%) & $43(69)$ & $51(77)$ & 0.31 & $61(79)$ & $79(81)$ & 0.82 \\
\hline Lesion length, mm & $23.5(13.7)$ & $31.9(21.1)$ & 0.02 & $28.7(14.8)$ & $37.6(19.6)$ & $<0.001$ \\
\hline
\end{tabular}

$\mathrm{CP}$, calcified plaque; HRP, high risk plaque; LD-NCP, low-density non-calcified plaque; NCP, non-calcified plaque; \%PAV, percent atheroma volume; PR, positive remodelling ; PV, plaque volume.

Table 4 Per-lesion adverse plaque characteristics by artificial intelligence enabled quantitative coronary computed tomographic angiography by non-obstructive versus obstructive angiographic stenosis stratified by age

\begin{tabular}{|c|c|c|c|c|c|c|}
\hline \multirow[b]{2}{*}{ Variable } & \multicolumn{3}{|c|}{ Non-obstructive $(<50 \%)$ per-lesion } & \multicolumn{3}{|c|}{ Obstructive $(\geq 50 \%)$ per-lesion } \\
\hline & $\begin{array}{l}\text { Age }<65 \\
(\mathrm{~N}=71)\end{array}$ & $\begin{array}{l}\text { Age } \geq 65 \\
(N=116)\end{array}$ & $P$ value & $\begin{array}{l}\text { Age <65 } \\
(\mathrm{N}=77)\end{array}$ & $\begin{array}{l}\text { Age } \geq 65 \\
(\mathrm{~N}=98)\end{array}$ & $P$ value \\
\hline $\mathrm{PV}, \mathrm{mm}^{3}$ & $60.5(79.8)$ & $105.6(140.5)$ & 0.001 & $103.8(134.5)$ & $109.7(131.4)$ & 0.83 \\
\hline LD-NCP, mm ${ }^{3}$ & $1.6(3.1)$ & $2.0(6.6)$ & 0.56 & $4.2(8.2)$ & $2.6(10.0)$ & 0.26 \\
\hline $\mathrm{NCP}, \mathrm{mm}^{3}$ & $38.4(50.8)$ & $56.2(75.8)$ & 0.058 & $74.6(108.1)$ & $61.5(85.8)$ & 0.33 \\
\hline $\mathrm{CP}, \mathrm{mm}^{3}$ & $22.1(39.1)$ & $49.4(86.8)$ & 0.004 & $29.2(51.5)$ & $48.2(72.7)$ & 0.04 \\
\hline PAV (total plaque) & $41.0(17.4)$ & $44.8(16.8)$ & 0.17 & $49.9(18.6)$ & $54.7(16.5)$ & 0.07 \\
\hline \% PAV (LD-NCP) & $1.5(3.3)$ & $0.7(1.3)$ & 0.038 & $2.0(3.0)$ & $1.2(2.8)$ & 0.12 \\
\hline$\%$ PAV (NCP) & $25.6(12.4)$ & $25.2(12.9)$ & 0.81 & $33.1(19.3)$ & $30.5(15.4)$ & 0.32 \\
\hline$\%$ PAV (CP) & $13.7(15.7)$ & $18.6(18.1)$ & 0.07 & $13.9(15.6)$ & $30.5(15.4)$ & 0.001 \\
\hline$\%$ Plaque calcified & $28.0(28.5)$ & $36.8(28.9)$ & 0.06 & $27.3(26.8)$ & $38.2(27.3)$ & 0.007 \\
\hline Remodelling index & $1.13(0.20)$ & $1.06(0.21)$ & 0.02 & $1.10(0.28)$ & $1.05(0.28)$ & 0.24 \\
\hline Positive remodeling $>1.1$ & $29(41 \%)$ & $34(29 \%)$ & 0.36 & $25(33 \%)$ & $29(30 \%)$ & 0.78 \\
\hline Intermediate remodelling & $28(39 \%)$ & $46(40 \%)$ & & $26(34 \%)$ & $27(28 \%)$ & \\
\hline Negative remodelling & $14(20 \%)$ & $36(31 \%)$ & & $26(34 \%)$ & $42(43 \%)$ & \\
\hline HRP (LD-NCP +PR) & $34(48 \%)$ & $42(36 \%)$ & 0.16 & $31(40 \%)$ & $33(34 \%)$ & 0.40 \\
\hline Lesion length, $\mathrm{mm}$ & $13.9(10.4)$ & $18.1(15.6)$ & 0.02 & $18.3(15.5)$ & $18.2(15.1)$ & 0.90 \\
\hline
\end{tabular}

$\mathrm{CP}$, calcified plaque; HRP, high risk plaque; LD-NCP, low-density non-calcified plaque; NCP, non-calcified plaque; PAV, per cent atheroma volume; PR, positive remodelling ; PV, plaque volume. 
near significantly higher volume of NCP in age $<65$ (38.4 $\mathrm{mm}^{3}$ vs $\left.56.2 \mathrm{~mm} 3 ; \mathrm{p}<0.058\right)$, as well as a higher remodelling index (1.13 vs $1.06 ; \mathrm{p}=0.02)$ with shorter lesion length $(13.9 \mathrm{~mm}$ vs $18.1 \mathrm{~mm} ; \mathrm{p}=0.02)$. An illustrative case of obstructive stenosis in a 39-year-old with high NCP and LD-NCP burden is shown in figure 1. An illustrative case of non-obstructive stenosis in a 55-year-old with high NCP is shown in figure 2. Age $>65$ patients with non-obstructive lesions exhibit higher calcified plaque $\left(49.4 \mathrm{~mm}^{3}\right.$ vs 22.1 $\left.\mathrm{mm}^{3} ; \mathrm{p}=0.004\right)$ and near significant $\%$ plaque calcified (36.8\% vs $28.0 \%$; $p=0.06$ ) compared with patient $<65$. An illustrative case of calcified plaque in a 74-year-old with high calcified burden is shown in figure 3 .

Online supplemental appendix $\mathrm{C}$ shows per-patient and per-lesion analysis within the younger and older age cohorts for non-obstructive and obstructive stenoses. In both younger and older individuals, PV and CP are higher for obstructive stenosis versus non-obstructive stenosis. In older adults, there was no difference between the volume of LD-NCP in non-obstructive and obstructive lesions, while calcified plaque was significantly higher in obstructive versus non-obstructive lesions $\left(366.5 \mathrm{~mm}^{3}\right.$ vs $224 \mathrm{~mm}^{3}$; $\mathrm{p}=0.01$ ).

\section{DISCUSSION}

In this analysis of CREDENCE, we uniquely applied AI for quantitative plaque evaluation and add several important new observations to the expanding body of literature on APC identified by CCTA. Our study found that APCs of high-grade stenoses differ by age with patients $<65$ years exhibiting greater PV, LD-NCP, NCP and lesion length in obstructive lesions compared with non-obstructive lesions. Patients $>65$ years exhibited a greater burden of calcified plaque in both non-obstructive and obstructive stenoses. Furthermore, in patients $>65$ with obstructive stenosis, while there was a higher calcified and NCP burden, younger patients had similar LD-NCP as older patients with obstructive lesions.

Recent validation of the AI-guided approach applied in this study allows for accurate AI evaluation of stenosis as well allowing for quantification of PV to an order of $3-5 \mathrm{~mm}^{3}$ that may not be readily identified by expert readers. ${ }^{12} 1819$ This data extends atherosclerosis evaluation beyond previous logistic regression models incorporating plaque as one of many variables defined by manual methods. ${ }^{20}$ In addition, the use of well-validated deep machine learning approaches allows for enhanced and rapid image processing. Previous validation data from our group has shown that this AI approach can perform whole heart atherosclerosis and stenosis evaluation in approximately $10 \mathrm{~min}$, whereas manual approaches may take several hours to complete and be prone to variability.

Through this AI approach, we show that atherosclerosis in younger adults with non-obstructive stenoses demonstrate comparable plaque characterisation when compared with older adults. This includes the presence of high-risk plaque features such as NCP, LD-NCP and
PR that qualitatively portend, by SCCT expert consensus, a future risk of cardiovascular events. ${ }^{21}$ Importantly, AI identified a higher PAV of LD-NCP in younger individuals with non-obstructive atherosclerosis, a plaque type identified as highest risk by cardiac CT. ${ }^{22}$ These data affirm prior findings that younger patients have high volumes of non-calcified atherosclerosis with a higher degree of LD-NCP, while older patients develop a higher degree of calcified plaque in both non-obstructive and obstructive lesions. ${ }^{22}$ Lowenstern $e t a l$ s subanalysis of the PROMISE (Prospective Multicenter Imaging Study for Evaluation of Chest Pain) study also supports the finding that older patients have more calcified plaque. ${ }^{23}$ However, importantly, the study also showed that high calcium scores did not confer risk for MACE in older patients while it did conferred risk for MACE in individuals younger than $65 .^{23}$ The study does not comment on additional APCs like LD-NCP, but remains consistent with our findings and re-emphasises the benefit of addressing plaque characteristics for prognostication for MACE at different ages. In our study, age stratification identified differences in overall plaque burden, which may help identify younger patients who may be at risk for future acute coronary syndrome (ACS) despite having non-obstructive stenoses.

While the literature on APCs and high-risk lesion assessment is rapidly expanding, there is still limited information regarding age-oriented plaque composition. In particular, identifying which younger adults are at risk for $\mathrm{CAD}$ remains challenging due to limitations in current risk calculators and insufficient data in this group. ${ }^{24}$ Additionally, limitations exist in identifying atherosclerosis via conventional approaches, such as stress testing, making younger adults less likely to be treated with preventive therapies. While Ruiz-García et al published a substudy of the PROSPECT (Prospective Natural History Study of Coronary Atherosclerosis) trial evaluating APCs of nonculprit lesions via intravascular ultrasound (IVUS) that concluded that patients older than 65 had greater plaque burden, necrotic core and dense calcium content than patients younger than 65 (regardless of gender),$^{25}$ this study involved non-culprit lesions in patients who had already experienced ACS and could not be generalised to a younger population in the preventive stages of disease.

In the CCTA literature, an age-based study was recently conducted by Conte et al who evaluated a subgroup of patients from the ICONIC (Incident Coronary Syndromes Identified by Computed Tomography) trial to determine whether APCs of culprit lesions varied by age and showed that older patients had greater total PV, specifically calcified plaque, as well as greater segment involvement and segment severity scores than younger patients. ${ }^{26}$ However, again, the study cohort was comprised of ACS patients and the lesion level analysis pertained specifically to culprit lesions, limiting the ability to generalise findings. Additionally, Kim et al initiated a substudy of the PARADIGM (Progression of AtheRosclerotic PlAque DetermIned by Computed TomoGraphic Angiography Imaging) trial, a cohort with suspected or known $\mathrm{CAD}$, and showed that 
the rate of plaque progression, specifically densely calcified plaque, increased with age. ${ }^{27}$ The study's intention was to identify gross trends in a middle-aged cohort ( $40-75$ years), and to that end, whole heart plaque was evaluated while lesion-based changes were unaddressed.

While calcified plaque increases with age, a finding consistent with our data, this does not account for the high risk ACPs at younger ages that may be important for early screening. ${ }^{24}{ }^{28}$ Our study findings suggest that the quantification of APCs associated within plaque identified by AI-QCT, namely, LD-NCP, NCP and PV and lesion length may also provide prognostic value when screening patients $<65$ years. Understanding this plaque phenotype also may allow for enhanced opportunities to understand and modulate the plaque phenotype through preventive therapies such as statins and newer agents such as PCSK-9 inhibition. ${ }^{29}$

This study is subject to limitations. While patients were enrolled prospectively from a large, multicentre clinical trial with evaluation by a blinded core laboratory, the evaluation was post hoc and not powered to detect differences in plaque types. About one-third of the patients had demonstrable atherosclerosis despite the absence of symptoms, which may not be fully representative of a stable chest pain population, but also represent a limitation in current guidelines for testing. Additionally, the CCTAs evaluated reflect a single point in time rather than a longitudinal period thereby limiting knowledge of plaque progression as reflected at various ages. Though coronary lesions are dynamic, changes in PV composition as a function of worsening stenosis severity was not evaluated. Finally, we used angiographic coronary stenosis as a marker of CAD severity. While large-scale prognosis of AI-QCT defined quantitative plaque composition has not yet been performed, it remains the subject of future study in the upcoming CONFIRM2 study (Coronary CT Angiography Evaluation for Clinical Outcomes: An International Multicenter Registry; NCT04279496).

In sum, AI-QCT identifies a unique APC signature that differs by age with patients $<65$ years exhibiting greater $\mathrm{PV}$, LD-NCP, NCP and lesion length in obstructive compared with non-obstructive lesions. While no difference in plaque composition was observed in obstructive stenoses for patients $>65$ years, older patients had greater CP volume and patients $<65$ had more NCP and LD-NCP. These findings have important implications for prognosticating younger and older adults with unique plaque phenotypes identified by AI-QCT that allows a foundation for AI-guided precision approaches to cardiovascular prevention.

\footnotetext{
Author affiliations

${ }^{1}$ Thomas Jefferson University Hospital, Philadelphia, Pennsylvania, USA

${ }^{2}$ Cleerly Health, New York, New York, USA

${ }^{3}$ UNICA, Unit of Cardiovascular Imaging, CHRC Campus Nova Medical School, Lisboa, Portugal

${ }^{4}$ Cardiology, Yonsei University Health System, Seodaemun-gu, Seoul, Korea

${ }^{5}$ Ontact Health, Inc, Seoul, Korea

${ }^{6}$ Department of Medicine, Inje University Ilsan Paik Hospital, Goyang, Korea

${ }^{7}$ Cardiology, Kangwon National University Hospital, Chuncheon, Kangwon, Korea
}

${ }^{8}$ Department of Internal Medicine, Seoul National University Hospital, Jongno-gu, Seoul, Korea

${ }^{9}$ Cardiovascular Center, Keimyung University Dongsan Hospital, Daegu, Korea

${ }^{10}$ Division of Cardiology, Department of Internal Medicine, Catholic Kwandong

University International Saint Mary's Hospital, Incheon, Korea (the Republic of)

${ }^{11}$ Cardiology, Ewha Women's University Mokdong Hospital, Seoul, Korea

${ }^{12}$ Mobile Cardiology Associates, Mobile, Alabama, USA

${ }^{13}$ Department of Imaging, Fondazione Toscana Gabriele Monasterio, Pisa, Italy

${ }^{14}$ Cardiac Center of Texas, McKinney, Texas, USA

${ }^{15}$ Department of Radiology, Fuwai Hospital State Key Laboratory of Cardiovascular

Disease, Beijing, China

${ }^{16}$ Fuwai Hospital State Key Laboratory of Cardiovascular Disease, Beijing, China

${ }^{17}$ Houston Methodist Hospital, Houston, Texas, USA

${ }^{18}$ Cardiovascular Center, Saint Luke's International Hospital, Chuo-ku, Tokyo, Japan

${ }^{19}$ Department of Radiology and Radiological Science, Medical University of South

Carolina, Charleston, South Carolina, USA

${ }^{20}$ VU University Medical Centre Amsterdam, Amsterdam, Noord-Holland,

Netherlands

${ }^{21}$ Department of Cardiology, Vrije Universiteit Amsterdam, Amsterdam, Netherlands

${ }^{22}$ Saint Luke's Mid America Heart Institute, Kansas City, Missouri, USA

${ }^{23}$ Cardiology, Kaiser Permanente, San Jose, California, USA

${ }^{24}$ Heart Center Research, Huntsville, Alabama, USA

${ }^{25}$ Renown Health, Reno, Nevada, USA

${ }^{26}$ Oconee Heart and Vascular Center, Saint Marys Medical Group, Athens, Georgia, USA

${ }^{27}$ Division of Cardiology, Hopital du Sacre-Coeur de Montreal, Montreal, Québec, Canada

${ }^{28}$ Cardiology, VU University Medical Centre Amsterdam, Amsterdam, Noord-Holland, Netherlands

${ }^{29}$ Centro Cardiologico Monzino Istituto di Ricovero e Cura a Carattere Scientifico, Milano, Lombardia, Italy

${ }^{30}$ Cardiology, Houston Methodist Hospital, Houston, Texas, USA

${ }^{31}$ Medicine (Cardiology), University of Virginia Health System, Charlottesville, Virginia, USA

${ }^{32}$ Division of Cardiology and Department of Radiology, The George Washington University School of Medicine and Health Sciences, Washington, District of Columbia, USA

\section{Twitter Andrew D Choi @AChoiHeart}

Contributors JE, RJe and JKM contributed to blinded core imaging core laboratory analysis and statistical support as requested by the study authors. RJo, ADC and TRC evaluated statistical methodology. RJo, JPE (prior to employment by Cleerly) and ADC conceived and designed the analysis, performed the analysis, wrote the paper and evaluated methodology. All other authors provided acquisition of study data as well as critical revision of the manuscript for important intellectual content. This study was an investigator initiated study.

Funding ADC is supported by a grant from the GW Heart and Vascular Institute.

Competing interests Equity Interest Cleerly, JE, HM, ADC, Employees of Cleerly RJe, TRC, JKM, JE.

Patient consent for publication Not applicable.

Ethics approval The institutional review board of each enrolling site approved the study protocol and all patients provided written informed consent.

Provenance and peer review Not commissioned; internally peer reviewed.

Data availability statement Data may be obtained from a third party and are not publicly available. Index data for the CREDENCE trial has been previously published. De-identified patient data are not publicly available, except if necessary to confirm study results; requests for data may be made by contacting Dr James Min (James. Min@cleerlyhealth.com).

Open access This is an open access article distributed in accordance with the Creative Commons Attribution Non Commercial (CC BY-NC 4.0) license, which permits others to distribute, remix, adapt, build upon this work non-commercially, and license their derivative works on different terms, provided the original work is properly cited, appropriate credit is given, any changes made indicated, and the use is non-commercial. See: http://creativecommons.org/licenses/by-nc/4.0/.

ORCID iDs

Rebecca Jonas http://orcid.org/0000-0003-0203-6210 


\section{REFERENCES}

1 Marwick TH, Cho I, Ó Hartaigh B, et al. Finding the gatekeeper to the cardiac catheterization laboratory: coronary CT angiography or stress testing? J Am Coll Cardiol 2015;65:2747-56.

2 Budoff MJ, Dowe D, Jollis JG, et al. Diagnostic performance of 64-multidetector row coronary computed tomographic angiography for evaluation of coronary artery stenosis in individuals without known coronary artery disease: results from the prospective multicenter accuracy (assessment by coronary computed tomographic angiography of individuals undergoing invasive coronary angiography) trial. J Am Coll Cardiol 2008;52:1724-32.

3 Abdelrahman KM, Chen MY, Dey AK, et al. Coronary computed tomography angiography from clinical uses to emerging technologies: JACC state-of-the-art review. J Am Coll Cardiol 2020;76:1226-43.

4 Singh G, Al'Aref SJ, Van Assen M, et al. Machine learning in cardiac CT: basic concepts and contemporary data. J Cardiovasc Comput Tomogr 2018;12:192-201.

5 Puchner SB, Liu T, Mayrhofer T, et al. High-risk plaque detected on coronary CT angiography predicts acute coronary syndromes independent of significant stenosis in acute chest pain: results from the ROMICAT-II trial. J Am Coll Cardiol 2014;64:684-92.

6 Diaz-Zamudio M, Fuchs TA, Slomka P, et al. Quantitative plaque features from coronary computed tomography angiography to identify regional ischemia by myocardial perfusion imaging. Eur Heart J Cardiovasc Imaging 2017;18:499-507.

7 Stuiffzand WJ, van Rosendael AR, Lin FY, et al. Stress myocardial perfusion imaging vs coronary computed tomographic angiography for diagnosis of invasive Vessel-Specific coronary physiology: predictive modeling results from the computed tomographic evaluation of atherosclerotic determinants of myocardial ischemia (CREDENCE) trial. JAMA Cardiol 2020;5:1338-48.

8 Nerlekar N, Ha FJ, Cheshire C, et al. Computed tomographic coronary Angiography-Derived plaque characteristics predict major adverse cardiovascular events: a systematic review and metaanalysis. Circ Cardiovasc Imaging 2018;11:e006973.

9 Feuchtner G, Kerber J, Burghard P, et al. The high-risk criteria lowattenuation plaque $<60 \mathrm{HU}$ and the napkin-ring sign are the most powerful predictors of MACE: a long-term follow-up study. Eur Heart J Cardiovasc Imaging 2017;18:772-9.

10 Rizvi A, Hartaigh Bríain Ó, Knaapen P, et al. Rationale and design of the CREDENCE trial: computed tomographic evaluation of atherosclerotic determinants of myocardial ischemia. BMC Cardiovasc Disord 2016;16:190.

11 Abbara S, Blanke P, Maroules CD, et al. SCCT guidelines for the performance and acquisition of coronary computed tomographic angiography: a report of the Society of Cardiovascular Computed Tomography Guidelines Committee: endorsed by the North American Society for Cardiovascular Imaging (NASCI). J Cardiovasc Comput Tomogr 2016;10:435-49.

12 United States Food and Drug Administration. Cleerly Labs 510 (K) premarket notification, 2019. Available: https://www.accessdata.fda. gov/cdrh docs/pdf19/K190868.pdf

13 Choi AD, Marques H, Kumar V. CT evaluation by artificial intelligence for atherosclerosis, stenosis and vascular morphology (CLARIFY): a multi-center, international study. J Cardiovasc Comput Tomogr 2021;15:470-6.
14 Leipsic J, Abbara S, Achenbach S, et al. SCCT guidelines for the interpretation and reporting of coronary CT angiography: a report of the Society of cardiovascular computed tomography guidelines Committee. J Cardiovasc Comput Tomogr 2014;8:342-58.

15 Nakazato R, Shalev A, Doh J-H, et al. Quantification and characterisation of coronary artery plaque volume and adverse plaque features by coronary computed tomographic angiography: a direct comparison to intravascular ultrasound. Eur Radiol 2013;23:2109-17.

16 Rizvi A, Hartaigh Briain Ó, Danad I, et al. Diffuse coronary artery disease among other atherosclerotic plaque characteristics by coronary computed tomography angiography for predicting coronary vessel-specific ischemia by fractional flow reserve. Atherosclerosis 2017;258:145-51.

17 Danad I, Raijmakers PG, Driessen RS, et al. Comparison of coronary CT angiography, SPECT, PET, and hybrid imaging for diagnosis of ischemic heart disease determined by fractional flow reserve. JAMA Cardiol 2017;2:1100-7.

18 Choi AD, Marques H, Kumar V, et al. CT evaluation by artificial intelligence for atherosclerosis, stenosis and vascular morphology (clarify): a multi-center, internaltional study. J Cardiovasc Comput Tomogr 2021.

19 Choi AD, Blankstein R. Becoming an expert practitioner: the lifelong journey of education in cardiovascular imaging. JACC Cardiovasc Imaging 2021;14:1594-7.

20 Al'Aref SJ, Maliakal G, Singh G, et al. Machine learning of clinical variables and coronary artery calcium scoring for the prediction of obstructive coronary artery disease on coronary computed tomography angiography: analysis from the CONFIRM registry. Eur Heart J 2020;41:359-67.

21 Shaw LJ, Blankstein R, Bax JJ, et al. Society of Cardiovascular Computed Tomography / North American Society of Cardiovascular Imaging - Expert Consensus Document on Coronary CT Imaging of Atherosclerotic Plaque. J Cardiovasc Comput Tomogr 2021;15:93-109.

22 Schmermund A, Schwartz RS, Adamzik M, et al. Coronary atherosclerosis in unheralded sudden coronary death under age 50: histo-pathologic comparison with 'healthy' subjects dying out of hospital. Atherosclerosis 2001;155:499-508.

23 Lowenstern A, Alexander KP, Hill CL, et al. Age-related differences in the noninvasive evaluation for possible coronary artery disease: insights from the prospective multicenter imaging study for evaluation of chest pain (PROMISE) trial. JAMA Cardiol 2020;5:193-201

24 Michos ED, Choi AD. Coronary artery disease in young adults: a hard lesson but a good teacher. J Am Coll Cardiol 2019;74:1879-82.

25 Ruiz-García J, Lerman A, Weisz G, et al. Age- and gender-related changes in plaque composition in patients with acute coronary syndrome: the PROSPECT study. Eurolntervention 2012;8:929-38.

26 Conte E, Dwivedi A, Mushtaq S, et al. Age- and sex-related features of atherosclerosis from coronary computed tomography angiography in patients prior to acute coronary syndrome: results from the ICONIC study. Eur Heart J Cardiovasc Imaging 2021;22:24-33.

27 Kim M, Lee S-P, Kwak S, et al. Impact of age on coronary artery plaque progression and clinical outcome: a paradigm substudy. $J$ Cardiovasc Comput Tomogr 2021:15:232-9.

28 Singh A, Collins BL, Gupta A, et al. Cardiovascular Risk and Statin Eligibility of Young Adults After an MI: Partners YOUNG-MI Registry. J Am Coll Cardiol 2018;71:292-302.

29 Lee S-E, Chang H-J, Sung JM, et al. Effects of Statins on Coronary Atherosclerotic Plaques: The PARADIGM Study. JACC Cardiovasc Imaging 2018;11:1475-84. 